\title{
Elaboración de modelos anatómicos mediante técnicas de plastinación como alternativa al uso de animales en docencia y como recurso esencial en el proceso de enseñanza- aprendizaje.
}

\section{Development of anatomic models through plastination techniques as an alternative to the use of animals in teaching and as an essential resource in the teaching-learning process}

\author{
Ana M. Molina López*, Andrés Diz Plaza ${ }^{+}$, Nahúm Ayala Soldado, Lorena Diz \\ Conde, Antonio Lora Benítez, Alfonso Martínez Galisteo, Joaquin Vivo \\ Rodríquez, Jose Miguel Navas Lloret, María I Barasona Carmona, Francisca \\ Bujalance Reyes, Andrés Rueda Jiménez, Ana Fernández Díez, Estrella \\ Montero Pérez-Barquero, y Rosario Moyano Salvago
}

Cómo citar este artículo:

Molina, A., Diz, A. et al. (2019). Elaboración de modelos anatómicos mediante técnicas de plastinación como alternativa al uso de animales en docencia y como recurso esencial en el proceso de enseñanzaaprendizaje. Revista de Innovación y Buenas Prácticas Docentes, 8(4), 24-32.

\section{Resumen:}

La implantación del principio de las 3Rs (Reducción, Refinamiento y Reemplazo), descritas por Russell y Burch en 1959, fue el punto de partida de numerosos cambios en la utilización de los animales, tanto en el ámbito de la investigación como de la docencia. Por tanto, es necesario ir propiciando el empleo de alternativas y que el profesorado disponga de recursos y de una buena información. Este proyecto se propone como objetivo esencial la elaboración de modelos animales mediante técnicas de plastinación como recurso docente alternativo al uso de animales vivos, y proporcionando al profesorado la disposición de medios y recursos esenciales en el proceso de enseñanza-aprendizaje del alumnado.

Los alumnos podrán disponer de una variedad de modelos animales en las sesiones prácticas, y/o teórico/prácticas de las distintas disciplinas en las que sea necesario el uso de animales. Podrán trabajar con un modelo fiel al original sin necesidad de utilizar modelos vivos y podrán desarrollar las distintas técnicas y procedimientos que realizarían in vivo sin necesidad de generar un estrés innecesario a los animales por su manejo por personal aún no capacitado para ello, evitando situaciones que hagan peligrar el bienestar animal (RD 53/2003). El desarrollo de estos modelos plastinados supondrá recurso docente esencial para la adquisición de las habilidades específicas en distintas disciplinas Biomédicas que conlleven el uso de animales, tales como los Grados en Veterinaria, Biología, Medicina, Enfermería, etc.

Palabras clave: modelos animales, plastinación; métodos alternativos; animales; principio 3Rs. 


\section{Abstract:}

The implementation of the 3Rs principles (Reduction, Refinement and Replacement), described by Russell and Burch in 1959, was the starting point of numerous changes in the use of animals in the fields of research and teaching. Therefore, it is necessary to foster the use of alternative methods and provide teachers with resources and good information. This project aims to develop animal models through the application of plastination techniques as an alternative teaching resource to the use of animals. In this way, the project aims to provide teaching staff with tools and resources that are essential in the teachinglearning process.

The students will be able to use a variety of animal models in practical and/or theoretical-practical sessions of the different subject areas, in which the use of animals is necessary. They will be able to work with animal models that are similar to the original, while avoiding the use of animals. Moreover, they will be able to learn and practice different techniques and procedures that they would carry out in vivo, without generating unnecessary stress to the animals caused by their management by untrained personnel. This approach is therefore in full conformity with existing animal welfare regulations (i.e. RD 53/2003). Plastinated models will become a an essential teaching resource for the acquisition of the specific skills in different Biomedical and Science disciplines that involve the use of animals, such as Veterinary Science, Biology, Environmental Sciences, Medicine, Nursing, etc.

Key Words: animal models; plastination; alternative methods; animals; 3Rs principles 


\section{INTRODUCCIÓN}

La implantación del principio de las 3 Rs (Reducción, Refinamiento y Reemplazo), descritas por Russell y Burch en 1959, fue el punto de partida de numerosos cambios en la utilización de los animales, tanto en el ámbito de la investigación como de la docencia. Ya en el artículo 24 de la Convenio Europeo para la Protección de los Animales Vertebrados utilizados en Experimentación y otros Fines Científicos se especifica: "aquellos procedimientos llevados a cabo con fines educativos o de entrenamiento se deben restringir a los absolutamente necesarios para los fines relativos a la enseñanza y el entrenamiento y se permitirán únicamente si sus objetivos no pueden ser conseguidos por métodos audiovisuales $u$ otros que sean suficientemente efectivos" (Convenio $\mathrm{n}^{\circ} 123$ del Consejo de Europa, Estrasburgo, 18 de marzo de 1986; BOE n‥ 256/1990, 25 de octubre de 1990). Es indudable que existe un gran número de métodos alternativos para ser utilizados en la docencia (modelos anatómicos, películas y videos, simulaciones de ordenador, material procedente de mataderos, estudios in vitro con líneas celulares, etc.) y que se dispone de información sobre ellos en bases de datos como Norwegian Inventory of Alternatives (NORINA) o European Resource Center for Alternatives in High Education (EURCA), pero aún es elevado el número animales de laboratorio, como ratas, ratones, conejos, etc. que se emplean para el desarrollo de las habilidades de los alumnos. Según el último informe, se calcula que solamente en la Unión Europea se utilizan unos 179.000 animales de laboratorio con fines docentes, representando el $1 \%$ del total de animales utilizados en investigación. Por tanto, es necesario ir propiciando el empleo de estas alternativas y que el profesorado disponga de recursos y de una buena información.

Diferentes estudios han demostrado el grado de satisfacción de los estudiantes con el empleo de métodos alternativos al uso de animales y, sin considerar los aspectos éticos, cómo los resultados académicos mejoraban considerablemente (Martínez, 2016; Latorre et al., 2016; Nguyen et al., 2019). En el apartado 2 del artículo 158 del Título V de los Estatutos de la UCO, se recoge entre los objetivos del Programa para el Desarrollo y Control de la Docencia, la Investigación y la Gestión, "promover proyectos docentes encaminados a una mejora de la calidad de la docencia", pues sin duda esto supone el desarrollo de un recurso docente esencial para la adquisición de las habilidades específicas en distintas disciplinas Biomédicas y de Ciencias de las titulaciones oficiales que imparte Universidad de Córdoba que conllevan el uso de animales.

Existen varias técnicas de conservación de cadáveres para poder apreciar las características anatómicas de los animales y comprender su función en la docencia práctica. Tradicionalmente, los Departamentos o Unidades de Anatomía y Embriología Veterinarias, para su docencia práctica, suelen utilizar material fresco (procedente de mataderos o de cadáveres obtenidos tras el sacrificio humanitario de animales domésticos), muy costoso debido a su poca duración en el tiempo (máximo 7-10 dias), o bien, lo más frecuente, material fijado. Para ello, los productos más frecuentemente utilizados por su calidad y bajo coste son el formaldehido como fijador y el fenol como antifúngico. Ambos productos están catalogados como altamente tóxicos y son muy volátiles, por lo que la legislación vigente es muy restrictiva en lo referente a los niveles ambientales permitidos para dichas sustancias, lo que obliga a realizar fuertes inversiones económicas en la instalación de sistemas de extracción adecuados para que los niveles ambientales de formaldehido y fenol sean los permitidos. Dados los problemas que presentan estos productos, contínuamente se han buscado alternativas para su sustitución por otros de menor toxicidad, siendo las técnicas de plastinación las que, hasta el momento, mejores resultados están aportando.

La técnica de plastinación (del término inglés plastination) presenta en la actualidad una gran difusión en todo el mundo dada la gran utilidad que viene 
demostrando para la docencia e investigación tanto en Anatomía y Embriología, como en otras disciplinas Veterinarias, Médicas o Biológicas, entre ellas Anatomía Patológica, Ginecología, Odontología, Parasitología, Radiología, Zoología, Botánica, etc. Asimismo, recientemente están mostrando gran interés en estas técnicas disciplinas del Área de Humanidades, como Arqueología (entre otras finalidades, para la conservación de pecios de madera muy antiguos y frágiles), Bellas Artes (desarrollo de modelos anatómicos para la obtención de moldes, esculturas, etc), lo que nos puede dar una idea del amplio campo de aplicación de dichas técnicas.

La plastinación se basa en la sustitución de los fluidos orgánicos (fundamentalmente el agua tisular y parte de las grasas) por siliconas, resinas de poliester o epoxis que permiten la conservación del material biológico eternamente, pudiendo ser almacenado sin necesidad de condiciones especiales y con la gran ventaja de ser inocuo y carente de toxicidad para quien lo manipula. Esta técnica supone, por tanto, una mayor calidad de las piezas obtenidas, secas, inodoras, atóxicas y con duración ilimitada, lo que permite emplearlas como modelos en docencia.

La Unidad Docente de Anatomía y Embriología Veterinarias de la UCO, es pionera en la instauración de éstas técnicas a nivel nacional y, aunque ha supuesto un enorme esfuerzo presupuestario poder adquirir las infraestructuras y productos necesarios para ello, desde el principio los resultados han sido muy satisfactorios. Además, dicha Unidad Docente dispone en sus instalaciones del Campus de Rabanales de un Museo Anatómico Veterinario (MAV-UCO) cuyo objetivo es facilitar al alumnado el estudio y comprensión de las disciplinas anatómicas al permitirle la observación de material anatómico expuesto adecuadamente y accesible en cualquier momento que lo desee, pues la experiencia nos demuestra que el alumno de Anatomía mejora ostensiblemente su formación cuando, además de libros de texto, apuntes, esquemas, vídeos, CD-Roms, etc., dispone de material anatómico a su alcance. Además, la implantación de Planes de Estudio acordes con el Espacio Europeo de Enseñanza Superior, exige la valoración en Créditos de las horas de estudio, y el Museo Anatómico se considera una pieza fundamental para el cumplimiento de estos objetivos.

Otro valor añadido de este material es que permite tanto al alumno como al profesional una formación continua directa, pues un Museo Anatómico bien estructurado, permite el estudio y sobre todo la aclaración de numerosas dudas que se plantean al consultar la bibliografía, pues ésta, aunque abundante y de calidad, siempre presenta ciertas limitaciones que pueden resolverse con la observación y análisis directo de preparaciones tangibles las cuales, expuestas adecuadamente, permiten visualizar las distintas estructuras presentes en las diferentes regiones y campos anatómicos.

Por todo lo expuesto anteriormente, las técnicas de plastinación, dadas sus claras ventajas respecto a otras, se han ido implantando en gran número de universidades a nivel mundial, reduciendose así el uso del formol en las sesiones prácticas de numerosas disciplinas que usan animales para su docencia. 


\section{OBJETIVOS}

El objetivo principal es la elaboración de modelos mediante técnicas de plastinación de diferentes animales (ratón, rata, cobaya y conejo) como un recurso docente para las disciplinas de Biociencias que se vienen impartiendo en las titulaciones oficiales de la Universidad de Córdoba que conlleven el uso de animales, procurando siempre su máxima difusión entre el profesorado. Para ello nos propusimos:

1. Aplicar la técnica de plastinación como alternativa al uso de animales, y proporcionar al profesorado la disponibilidad de estos medios y recursos esenciales en el proceso de enseñanza-aprendizaje del alumnado.

2. Disminuir paulatinamente los niveles de gases tóxicos (formol, fenol, etc.) utilizados en otro tipo de técnicas, incrementando el uso de material anatómico plastinado.

3. Facilitar al profesorado y alumnado el estudio de la Anatomía de diferentes especies animales mediante el uso de preparaciones anatómicas plastinadas de calidad.

4. Desarrollar una colección de material plastinado de calidad para ser expuesta permanentemente en el Museo Anatómico Veterinario de la UCO (MAV-UCO), que permita al estudiante el acceso directo a la misma durante el estudio.

\section{MATERIAL Y MÉTODOS}

Se realizó una selección de cadáveres de animales de la colonia de cría (ratón, rata, cobaya y conejo) de ambos sexos, del Servicio de Animales de Experimentación (SAEX) de la UCO, y se procesaron en el Laboratorio de Plastinación de la Unidad de Anatomía y Embriología Veterinarias del Departamento de Anatomía y A.P. Comparadas de la UCO, donde se procedió al desarrollo de diferentes modelos animales utilizando la técnica de plastinación con silicona. Los animales, excedentes de la colonia de cría, fueron previamente sacrificados en el Animalario, garantizándose el bienestar animal, y siguiendo en todo momento los métodos eutanásicos recomendados según especie en la normativa vigente (RD 53/2013).

\subsection{Protocolo de la técnica de plastinación (Técnica Biodur S-10)}

El protocolo de la Técnica Biodur S-10 (Von Hagens, 1986; De Jong\& Henrry, 2007) consiste en una serie de pasos que, descritos muy brevemente, son los siguientes:

- Fijación: hay varias técnicas dependiendo del material a plastinar, pero todas ellas tienen en común la utilización de formol a distintas concentraciones. En algunos casos, se puede suprimir o simultanear con el inicio de la deshidratación.

- Deshidratación y desengrasado: se realiza con pases sucesivos por acetona hasta que el nivel de agua residual es inferior al $2 \%$. Esta es la fase más costosa en lo referente a material fungible, pues son necesarias grandes cantidades de acetona.

- Impregnación forzada: es el paso más importante y delicado en esta técnica. Consiste en la sustitución de la acetona por polímeros (en nuestro caso silicona Biodur S-10 más elongador de cadenas (silicone chain elongator) Biodur S-3 al 1\%) mediante la disminución lenta y progresiva de la presión hasta llegar a 2-4 mbar. 
- Curación o polimerización: consiste en el endurecimiento de las preparaciones previamente impregnadas en silicona al ser estas expuestas a vapores de endurecedores volátiles, en éste caso Biodur S-6 (crosslinking agent o entrecruzador de cadenas). Durante esta fase, los órganos huecos pueden ser distendidos mediante la utilización de aire comprimido y debe realizarse en ambiente seco, por lo que se utiliza cloruro cálcico o sílica gel como absorbente de humedad. Es importante en esta fase posicionar adecuadamente la preparación anatómica para ver bien las estructuras a estudiar y obtener una preparación lo más estética posible.

- Almacenamiento: el material plastinado puede ser almacenado en estanterías o armarios para su uso en la docencia práctica o teórica, o montado en vitrinas de metacrilato o peanas cuando por su calidad se destine a ser expuesto en el MAV-UCO.

Siempre, como paso previo a la técnica de plastinación, se realizaron disecciones, repleciones vasculares con látex, silicona o resinas epoxi coloreadas para resaltar las estructuras vasculares, cortes anatómicos tomográficos, etc. Las técnicas anatómicas empleadas dependieron de las estructuras anatómicas que se pretendían observar y de la forma de presentación de las mismas.

\section{RESULTADOS Y DISCUSIÓN}

Se realizaron modelos animales mediante la técnica de plastinación de distintas especies de animales de experimentación (rata, ratón, cobaya y conejo), de diversa forma en función de lo que se pretendía estudiar:

a) Disecciones para el estudio del Aparato Locomotor, Sistemas Viscerales, Sistema Nervioso Central y Anatomía Topográfica (Figuras 1 y 2).

b) Cortes tomográficos en los planos transverso, sagital y dorsal (Figuras 3, 4 y 5).

De esta forma, en función de lo que se pretenda mostrar a los alumnos, dispondremos de un modelo fiel a la realidad. Son prototipos que pueden servir para realizar simulaciones de abordajes quirúrgicos, así como para aprender cómo se realiza el acceso a las distintas vías, sin necesidad del uso de animales vivos por parte de personal inexperto. Asimismo, los cortes tomográficos son de gran utilidad para el estudio de la anatomía topográfica y para ser comparados con imágenes obtenidas mediante técnicas de Tomografía Axial Computarizada (TAC) y Resonancia Magnética (RM).

Los alumnos podrán disponer de los distintos modelos plastinados en las sesiones prácticas y/o teórico/prácticas de las múltiples disciplinas en las que sea necesario el uso de animales. Podrán trabajar con un modelo fiel al original sin necesidad de utilizar modelos vivos. Además, podrán desarrollar las distintas técnicas y procedimientos que realizarían in vivo sin necesidad de generar estrés innecesario a los animales al ser manejados por personal inexperto, evitando situaciones que hagan peligrar el bienestar animal (de acuerdo con el Real Decreto 53/2013).

El desarrollo de estos modelos plastinados resultará un elemento fundamental en proceso de enseñanza-aprendizaje, como se desprende de las publicaciones de Bickley et al., 1987; Latorre et al., 2007; Frushtorfer et al., 2011; Sora et al., 2012 y 
Sora \& Jilavu, 2013, entre otras. Sin duda, esto supondrá el desarrollo de un recurso docente esencial para la adquisición de las habilidades específicas en distintas disciplinas Biomédicas que se imparten en las titulaciones oficiales de la Universidad de Córdoba y que conllevan el uso de animales, tales como los Grados en Veterinaria, Biología, Medicina, Enfermería, Fisioterapia, Ciencias del Medio Ambiente etc.

Por otro lado, entre otros, estos modelos plastinados seran expuestos en el MAV-UCO, por lo que ademas de lo anteriormente expuesto, ejercerán una labor divulgativa muy importante, ya que dichas instalaciones son visitadas tanto por docentes como por alumnos procedentes de centros de enseñanza ajenos a la UCO, tales como otras universidades, colegios, institutos, centros de formación profesional, etc. Finalmente, la experiencia nos permite afirmar que el material anatómico plastinado goza de gran aceptación por parte del alumnado y del profesorado, dada su inocuidad, facilidad de manejo, y la calidad estética de dichas preparaciones en comparación con las preparaciones conservadas en formol u otros fijadores y/o conservadores, coincidiendo con las conclusiones de Latorre et al., 2016 y Nguyen et al., 2019, entre otras publicaciones.

\section{CONCLUSIONES}

Este trabajo nos ha servido para mostrar al alumnado así como al profesorado, la posibilidad la aplicación del principio de las 3 Rs en la docencia de muy diversas titulaciones universitarias. Mediante la técnica de plastinación se pueden crear modelos de animales de laboratorio fieles a la realidad, que pueden ser utilizados como alternativa al uso de animales en docencia, reduciéndose así la utilización de los mismos por parte de personal inexperto, garantizándose la adquisición de mayores destrezas y, por tanto, preservando el bienestar animal en el momento que tenga que realizarse algún procedimiento in vivo.

En base a los resultados obtenidos, podemos afirmar que el uso de modelos plastinados puede reducir al mínimo la exposición a productos tóxicos como el formaldehido y el fenol por parte del alumnado y profesorado de centros universitarios, así como de centros de educación primaria, segundaria y de formación profesional. Asimismo, aunque las infraestructuras y productos químicos para su realización suponen una inversión relativamente alta, los resultados obtenidos y la aceptación general de estos modelos por parte del profesorado y alumnado, alientan su incorporación paulatina en centros docentes, con el fin suprimir a medio plazo los productos tóxicos en los laboratorios de prácticas y aulas.

Finalmente, sugerimos a las autoridades universitarias tanto de la UCO como de otras universidades españolas y del resto del mundo, la instalación de laboratorios de plastinación centralizados y con financiación propia, para la producción de material plastinado para la docencia de numerosas disciplinas básicas, optativas y/o obligatorias. La plastinación es una técnica de alta calidad (véanse las figuras 1 a 5) a un coste "asumible" por la mayoría de las universidades de los países desarrollados o en vías de desarrollo, aunque, eso sí, requiere mucho tiempo de dedicación, imaginación y paciencia.

\section{AGRADECIMIENTOS}

Los autores quieren agradecer al Departamento de Anatomía y Anatomía Patológica Comparadas, así como al Servicio de Animales de Experimentación de la Universidad de Córdoba, su colaboración desinteresada. 
Esta publicación surge del Proyecto 2017-1-3010 del Plan de Innovación y Buenas Prácticas Docentes 2016-2017 de la UCO.

\section{BIBLIOGRAFÍA}

Bickley, H.C., von Hagens, G., \& Townsend, F.M. (1981). An improved method for the preservation of teaching specimens. Arch Pathol Lab Med, 105,674-676.

Comisión Europea. (s.f.) EU Reference Laboratory for alternatives to animal testing. Recuperado de: https://eurl-ecvam.jrc.ec.europa.eu/

Convenio Europeo para la Protección de los Animales Vertebrados utilizados en Experimentación y otros Fines Científicos (Convenio no 123 del Consejo de Europa), 18 de marzo de 1986, Estrasburgo. Instrumento de Ratificación, Boletín Oficial del Estado no. 256, de 25 de octubre de 1990.

DeJong, K.,\& Henry, R.W. (2007). Silicone plastination of biological tissue: Coldtemperature Technique BiodurTM S10/S15 technique and products. J Int Soc Plastination, 22,2-14.

Diz, A., Rodríguez-Barbudo, M.V., Rivero, J.L.L., Galisteo, A.M., \& Conde-Pérez, A.J. (1993). La plastificación como técnica de conservación de material anatómico. An. Vet., 9-10, 49-56.

Fruhstorfer, B.H., Palmer, J., Brydges, S., \& Abrahams, P.H. (2011). The use of plastinated prosections for teaching anatomy - the view of medical students on the value of this learning resource. Clin Anat. 24,246-252.

Latorre, R.M., Garcia-Sanz, M.P., Moreno, M., Hernandez, F., Gil, F., López, O., Ayala, M.D., Ramirez, G., Vazquez, J.M., Arencibia, A., \& Henry, R.W. (2007). How useful is plastination in learning anatomy? $J$ Vet Med Educ. 34,172-176.

Latorre, R., Bainbridge, D., Tavernor, A., \& López Albors, O. J. (2016). Plastination in Anatomy Learning: An Experience at Cambridge University. Vet Med Educ. 43(3), 226-234.

Martínez, V. (2016) Animales "de Experimentación" con Fines Docentes: Valoración de la Percepción por un Colectivo de Estudiantes Universitarios (Trabajo de Fin de Máster, Máster en Bioética y Derecho). Universidad de Barcelona.

Nguyen, V.H., Pham, P.T., Joo, K. y Jeter, C.B. (2019). Dental students' and residents'opinions and performance of Anatomy learning via cadavers or plastinated specimens. J. Plastination 31 (1),6-13.

Norecopa. Plastinated Rat Dissection. https://norecopa.no/norina/plastinated-rat-dissection

Real Decreto 53/2013, de 1 de febrero de 2013, por el que se establecen las normas básicas aplicables para la protección de los animales utilizados en experimentación y otros fines científicos, incluyendo la docencia.

Red Española para el Desarrollo de Métodos Alternativos a la Experimentación Animal. Recuperado de: http://www.remanet.net/

Servicio de listas de distribución de la Comunidad Académica Española. Recuperado de: https://listserv.rediris.es/cgi-bin/wa?INDEX

Sora, M.C., Jilavu, R., \& Matusz, P. (2012). Computer aided three-dimensional reconstruction and modeling of the pelvis, by using plastinated cross sections, as a powerful tool for morphological investigations. Surg Radiol Anat. 34, 731736. 
Sora, M.C., \& Jilavu, R. (2013). Three-dimensional reconstruction of a female pelvis using plastinated cross-sections - Using plastination for 3D reconstruction. $J$ Plastination. 25(1), 22-27.

von Hagens, G., Tiedemann, K., \& Kriz, W. (1987). The current potential of plastination. Anat Embryol, 175, 411-421.

von Hagens, G. (1986). Heidelberg Plastination Folder: Collection of technical leaflets for plastination. Heidelberg: Biodur Products. 